\title{
Áreas de recarga hídrica potencial en las microcuencas del distrito de Cachí, Cartago, Costa Rica
}

\author{
Pablo Ramírez Granados ${ }^{1,2}$, María Álvarez Jiménez ${ }^{2}$, José Castro Solís ${ }^{3}$ iD \& Ligia Solís Torres ${ }^{4}$ iD \\ 1. Universidad de Costa Rica, Centro de Investigaciones en Ciencias Geológicas, San Pedro de Montes de Oca, Costa \\ Rica; pablo.ramirez@ucr.ac.cr \\ 2. Universidad Nacional, Laboratorio de Hidrogeología y Manejo de Recursos Hídricos, Escuela de Ciencias \\ Ambientales, Heredia, Costa Rica. maria.alvarez.jimenez@una.cr \\ 3. Consultor Ambiental, Costa Rica. jacastros@gmail.com \\ 4. Universidad Nacional, Escuela de Química, Heredia, Costa Rica; ligia.solis.torres@una.cr
}

\author{
Recibido 10-VIII-2021 • Corregido 15-X-2021 • Aceptado 18-XI-2021 \\ DOI: https://doi.org/10.22458/urj.v13i2.3683
}

\begin{abstract}
Potential water recharge areas in the micro-basins of Cachí district, Cartago, Costa Rica." Introduction: Precipitation is the most important source in water recharge zones and to ensure its availability and management it is important to evaluate changes in land use. Objective: To determine the potential water recharge areas in six Cartago micro-basins. Methods: We determined hydrogeological units through gauges, and physical and hydraulic properties on the field. We used topographic curves to generate a digital elevation model and longitudinal profiles. Additionally, we produced a land-use change map and determined soil water balance. Results: The area presents good hydrogeological conditions in terms of springs, pinpointing the hydraulic connection of the units, and the soil favors water recharge in the upper parts. Above, where the springs are located, half of the land has forest cover, facilitating infiltration. The rest sustains agricultural activities $139 \%$ in the middle and lower parts of the basins). Forest areas present lower potential recharge values (approx. $<611,4 \mathrm{~mm})$ than areas of crops and pasture $(792,32$ $796,29 \mathrm{~mm})$. Conclusion: The site presents good hydrogeological conditions and areas of crops and pasture have higher potential recharge values than forests.
\end{abstract}

Keywords: Water resource, aquifer, land use.
RESUMEN. Introducción: La precipitación es la fuente más importante en las zonas recarga hídrica y para asegurar su disponibilidad y manejo es importante evaluar los cambios de uso de la tierra. Objetivo: Medir el potencial de recarga hídrica en seis microcuencas cartaginesas. Métodos: Determinamos unidades hidrogeológicas con galgas, y propiedades físicas e hidráulicas directamente en campo. Usamos curvas topográficas para generar un modelo de elevación digital y perfiles longitudinales. Además, produjimos un mapa de cambio de uso de la tierra y determinamos el balance hídrico del suelo. Resultados: El área presenta buenas condiciones hidrogeológicas en cuanto a manantiales, señalando la conexión hidráulica de las unidades, y el suelo favorece la recarga de agua en las partes altas. Arriba, donde se ubican los manantiales, la mitad del terreno tiene cobertura forestal, lo que facilita la infiltración. El resto sustenta actividades agrícolas (39\% en la parte media y baja de las cuencas). Las áreas forestales presentan menores valores de recarga potencial (aprox. $<611,4 \mathrm{~mm}$ ) que las áreas de cultivos y pastos (792,32-796,29mm). Conclusión: El sitio presenta buenas condiciones hidrogeológicas y las áreas de cultivos y pastos tienen valores de recarga potencial más altos que los bosques.

Palabras clave: Recurso hídrico, acuífero, utilización de la tierra. 
El agua subterránea es la principal fuente de agua para los diferentes usos; domésticos, agrícolas e industriales en el mundo, y aproximadamente un tercio de la población mundial utiliza el agua subterránea para consumo (Varol \& Davraz, 2015).

Es necesario asegurar en las zonas de recarga hídrica el flujo de agua manteniendo el uso de bosque como la cobertura más idónea en cuanto a la cantidad y calidad, así mismo es preciso comprender que el cambio de uso de la tierra puede influir en la dinámica de dicha recarga (Hall et al., 2020).

Debido a que el agua subterránea enfrenta una fuerte presión para cumplir con las demandas de consumo a futuro producto al acelerado crecimiento de la población, la expansión agrícola, la dinámica de los cambios de uso de la tierra y el cambio climático (Achu et al., 2020), este último afecta la recarga a través del aumento de la temperatura, los cambios en la evapotranspiración y la variabilidad de las precipitaciones (Kundzewicz \& Döll, 2009).

Paraíso, provincia de Cartago, distrito Cachí, se caracteriza por sus tierras con bosques pastos e importantes zonas de cultivos de legumbres, hortalizas y especialmente de café ubicados en la parte media y baja. Según Cortés, (2008) estas tierras son adecuadas para el cultivo de café con sombra y sin sombra.

Adicionalmente se desarrollan dos actividades importantes de acuerdo con el Instituto Costarricense de Turismo [ICT], (2002) el turismo por el potencial de la belleza del paisaje y la generación hidroeléctrica por la alta pluviometría la convierte en una de las áreas de mayor recarga de agua. De ella se abastece de agua potable tanto al Área Metropolitana de San José como a los cantones de Paraíso y Cartago (Cortés, 2008).

En este distrito se identificó una red de drenaje compuesta por seis microcuencas denominadas Guatuso, Urasca, Tambor-Naranjo, Zapote-Irola, Oro y Loáiza, por la dinámica y características del sitio es estudio fue necesario analizar la relación de las áreas de recarga y los usos de la tierra a través de un balance para conocer la disponibilidad del recurso hídrico. La recarga de agua se puede determinar por medio de varios métodos, y uno de ellos es el balance hídrico (Vélez \& Vásquez, 2004).

Nuestro estudio se enfoca en determinar las zonas de recarga potencial en el distrito de Cachí necesario para el manejo de las cuencas hidrográficas, desarrollo de las actividades productiva y la sostenibilidad ambiental del recurso hídrico local. 


\section{MATERIALES Y MÉTODOS}

Área de estudio: El distrito de Cachí se encuentra entre las coordenadas 517500 y 526500 m este y 10825000 y 1091 000m norte (Fig. 1.) del sistema de proyección Transversal de Mercator para Costa Rica (CRTM) y tiene un área de $41,24 \mathrm{~km}^{2}$. Constituye uno de los distritos del cantón de Paraíso y está conformado por las comunidades de San Jerónimo, Urasca, Peñas Blancas, Cachí, Volio, Loaiza y San Miguel. Alberga el embalse de Cachí el cual es utilizado para la generación hidroeléctrica y en él se desarrollan actividades agropecuarias y turísticas.

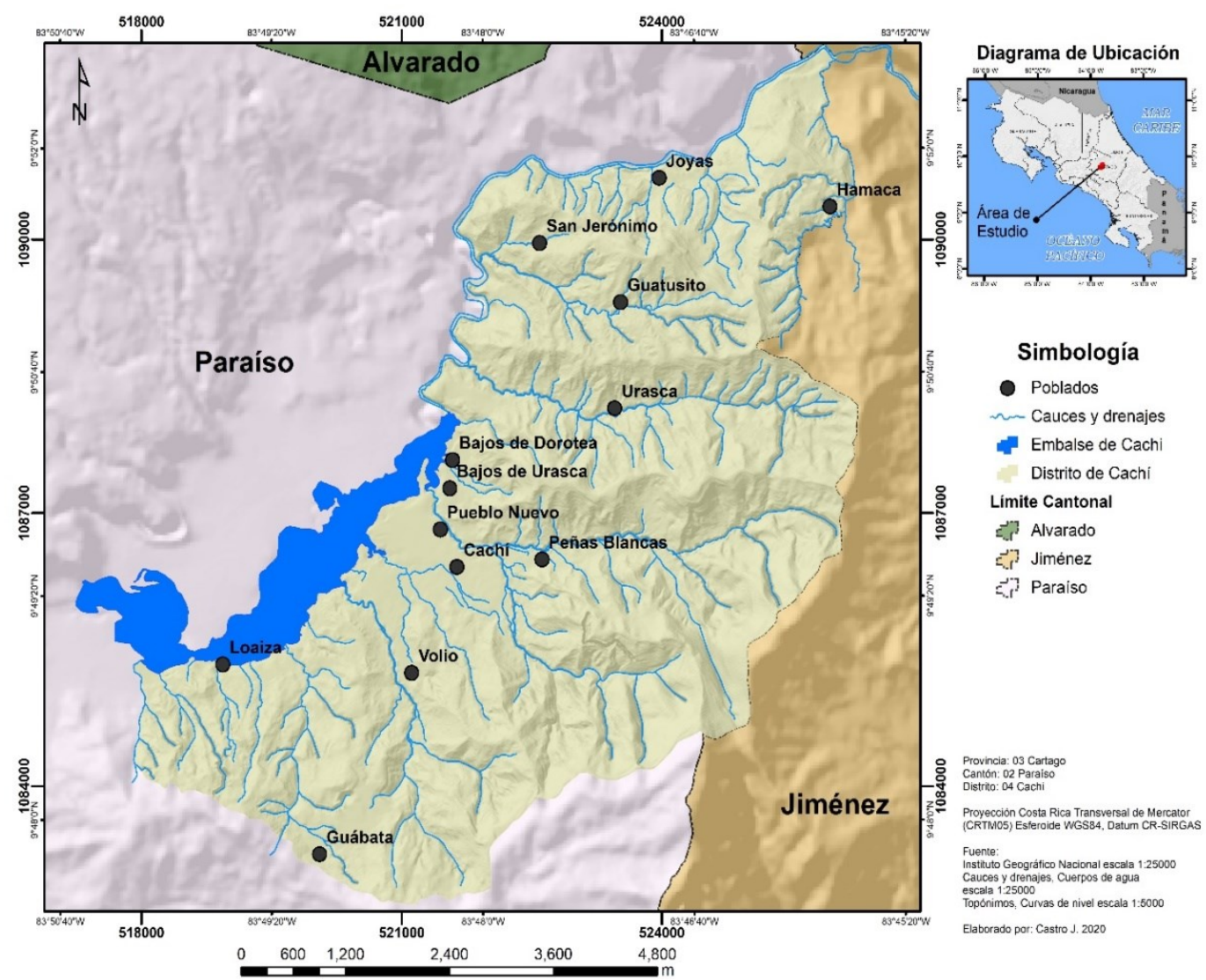

Fig. 1. Ubicación de las microcuencas del distrito de Cachí, Cartago

Análisis geomorfométrico: A partir de las curvas topográficas, escala 1:5000 (Acuña, 2008) del Instituto Geográfico Nacional (IGN) fue generado el Modelo de Elevación Digital (MED). Para la red de drenaje utilizando la capa de cauce y drenaje, escala 1:25 000 (Acuña, 2014). EI MED fue creado a través de la interpolación por Topo to Raster (Hutchinson, 1993) y a partir de él se obtuvo tres perfiles longitudinales.

Determinación de las unidades hidrogeológicas: Las unidades geológicas regionales y los aspectos estructurales se obtuvieron de Sojo et al. (2017). Con base en estas se realizó el trabajo de campo para establecer las unidades hidrogeológicas. La medición de la conexión hidráulica entre los sistemas acuíferos y la red de drenaje superficial fue realizada a partir de aforos diferenciales.

Para la construcción de la red de flujo de aguas subterráneas se consideró un acuífero libre con descargas por medio de manantiales, los cuales fueron considerados puntos específicos de 
carga hidráulica. La interpolación de las cargas hidráulicas se realizó con el método de función de base radial (Dressler, 2009).

Determinación de las propiedades físicas e hidráulicas de los materiales: Se seleccionó al azar 27 sitios distribuidos en el área de estudio de manera uniforme, dentro de la selección de cada uno se consideró unidades litológicas, tipo de suelo y uso de la tierra. Se realizó una calicata con un área de $1 \mathrm{~m}^{2}$ con una profundidad determinada por las raíces del sitio. Se ejecutaron pruebas de infiltración durante dos horas manteniendo una carga de agua inicial de $7,5 \mathrm{~cm}$ y una carga mínima de $2,5 \mathrm{~cm}$. Para cada uno de los sitios se obtuvo una muestra en núcleo de $10 \mathrm{~cm}$ de altura y $5 \mathrm{~cm}$ de diámetro, 2 muestras en núcleo de $1 \mathrm{~cm}$ de altura y $5 \mathrm{~cm}$ de diámetro y una muestra alterada de $2 \mathrm{~kg}$. Con ellas se determinaron las siguientes propiedades: densidad aparente, densidad real, granulometría, textura, retención de humedad y conductividad hidráulica.

Determinación del uso de la tierra: El uso de la tierra y la cobertura de la cuenca, fueron digitalizados a partir de la fotointerpretación de la imagen satelital de Planificación Regional y Urbana de la Gran Área Metropolitana [PRUGAM], (2007). Se digitalizaron vectores poligonales asignando a cada polígono una categoría específica. Para todos los polígonos fue revisada su geometría y topología.

Una vez obtenidos todos los polígonos de uso, se realizó corroboración de campo, utilizando un dispositivo Timble Juno 3D. La cantidad de puntos de control del uso de la tierra y la cobertura fue de 250 puntos en los sitios de acceso. Los usos fueron categorizados de forma detallada en agua, terreno descubierto, urbano/otros, pastos, cultivos y bosques. Para la aplicación en el balance hídrico, las categorías de los usos fueron en tres categorías: bosques, pastos y cultivos.

Cálculo del balance hídrico del suelo: Para determinar la recarga se empleó la metodología de Schosinsky (Schosinsky \& Losilla 2000, Schosinsky, 2006). Para la obtención del valor de precipitación mensual (P) en $\mathrm{mm}$, se utilizó el registro histórico de los valores de lluvia de la estación Cachí del Instituto Meteorológico Nacional (IMN). Para contabilizar la cantidad de agua que efectivamente llega al suelo y que infiltra se obtuvo el coeficiente de follaje (Cfo) y a partir de él, la retención del follaje (Ret).

Ec. 1

$$
\operatorname{Ret}=\left\{\begin{array}{c}
P \text { si } P \leq 5 \\
P \cdot C f o \text { si } P \cdot C f o \geq 5 \\
5 \text { si } P>5 \text { y } P \cdot C f o<5
\end{array}\right\}
$$

Schosinsky (2006) indica que el Cfo se toma como $20 \%$ como intercepción de la lluvia por follaje en bosque y un $12 \%$ para otras coberturas.

El coeficiente de infiltración (Ci) del agua está compuesto de tres componentes: la fracción que infiltra por efecto de la pendiente $(\mathrm{Kp})$, la fracción que infiltra por efecto de la vegetación (Kv) y la fracción que infiltra por efecto de la textura del suelo (Kfc):

Ec. 2

$$
C i=K p+K v+K f c \quad \text { con } \quad 0 \leq C i \leq 1
$$


Kfc puede tomar los siguientes valores:

Ec. 3

$$
K f c=\left\{\begin{array}{c}
0,267 \cdot \ln (f c)-0,000154 f c-0,723 \quad \text { si } 16<f c<1568 \\
\frac{0,0148 \cdot f c}{16} \quad \text { si } f c \leq 16 \\
1 \text { si } f c \geq 1568
\end{array}\right\}
$$

fc corresponde con la conductividad hidráulica saturada del suelo en los primeros $30 \mathrm{~cm}$ que se considera que está en contacto directo con la lluvia y fue obtenido por infiltrómetro de doble anillo.

La precipitación que infiltra $(\mathrm{Pi})$ en $\mathrm{mm}$ se calcula a continuación:

Ec. 4

$$
P i=C i \cdot(P-R e t)
$$

Seguidamente, la escorrentía superficial (Esc) en mm se determina:

Ec. 5

$$
E s c=P-R e t-P i
$$

Al inicio de un mes cualquiera, el suelo presenta una humedad inicial (Hsi) y la evapotranspiración es 0 . La condición de humedad a inicio de mes está dada por:

Ec. 6

$$
C 1=\frac{H s i-P M P+P i}{C C-P M P}
$$
humedad:

Una vez ocurrida la evapotranspiración, al final de mes, se obtiene una nueva condición de Ec. 7

$$
C 2=\frac{H s i-P M P+P i-(C 1 \cdot E P)}{C C-P M P}
$$

La humedad disponible (Hd) en $\mathrm{mm}$ se describe a continuación:

Ec. 8

$$
H d=H s i+P i-P M P
$$

Obtenido Hd se procedió a calcular Etr = evapotranspiración real $(\mathrm{mm})$ de la siguiente forma: Ec. 9

$$
E t r=\left\{\begin{array}{ll}
\frac{C 1+C 2}{2} \cdot E T & \text { si } \frac{C 1+C 2}{2} \leq H d \\
H d & \text { si } \frac{C 1+C 2}{2}>H d
\end{array}\right\}
$$


El valor de la humedad final del suelo (Hsf) a final de mes es dado por la ecuación:

Ec. 10

$$
H s f=\left\{\begin{array}{c}
H d+P m-E t r \quad \text { si } H d+P m-E t r<C C \\
C C \quad \text { si } H d+P m-E t r \geq C C
\end{array}\right\}
$$

El término Hsi $(\mathrm{mm})$ denominado humedad del suelo inicial, se determinó de la siguiente manera:

Ec. 11

$$
H s i=\left\{\begin{array}{c}
C C \\
H s f
\end{array}\right\}
$$

El valor perteneciente a Hsf corresponde con el valor del mes anterior, mientras que el valor de CC se relaciona con el valor de capacidad de campo. La selección del mes donde Hsi = CC se da cuando la secuencia de meses donde Pi es mayor que la evapotranspiración potencial (ETP) cambia.

Finalmente, el valor de recarga $(\mathrm{Rp})$ en $\mathrm{mm}$ fue calculado de la siguiente forma:

Ec. 12

$$
R p=P i+H s i-H s f-E t r
$$

Una vez determinadas las propiedades físicas e hidráulicas de los suelos, la lluvia, la pendiente y el uso de la tierra, fueron determinadas las zonas específicas para la aplicación del balance hídrico. La delimitación de las zonas se realizó utilizando como criterio la sobreposición de los datos anteriores, se identificaron las zonas específicas que presentaran cualidades particulares.

\section{RESULTADOS}

Geomorfometría: El sitio en estudio tiene variaciones topográficas que van desde los $11,4^{\circ}$ hasta los $88^{\circ}$, los drenajes superficiales presentan un recorrido en sentido este-oeste y sureste-sur. En las partes más altas presenta pendientes fuertes en un rango de $30^{\circ}-80^{\circ}$ ubicados en la formación Doán, cerca de los poblados Hamaca, Guatusito, Urasca y Peñas Blancas y en algunos sectores del cauce del río Reventazón en la Formación Aguacate.

En la parte sur del distrito, la formación Pacacua muestra un relieve más suave que se correlaciona con materiales sedimentarios provenientes de la deposición de materiales transportados que corresponden a los erosionados en las partes altas.

Hacia el norte es notable el estrangulamiento del río Reventazón por la zona de contacto entre la formación Aguacate y Cervantes. Así mismo en el sector del embalse y a lo largo de los tramos del río se evidencia niveles topográficos menores, en ciertos casos formando terrazas y que corresponden con depósitos recientes fluviales y lacustres (Fig. 2.).

El río Urasca tiene una variación topográfica con un descenso casi rectilíneo del río, desde los $1400 \mathrm{~m}$ hacia la formación Doán y continua constante sobre la formación Aguacate (Fig. 3.). Respecto al río Guatuso, el perfil presenta cambios altimétricos (entre $1400 \mathrm{~m}$ hasta $1200 \mathrm{~m}$ ), regulares en el trayecto sobre la Formación Doán, luego se observa un cambio abrupto en el gradiente en el contacto con la Formación Aguacate, entre ambas unidades podría darse la surgencia de agua considerando que la Formación Doán da origen al Acuífero Peñas Blancas. (Fig.4.).

Para el caso del río Naranjo (Fig. 5.), el perfil muestra un descenso fuerte relacionado a una mayor resistencia de los materiales que conforman la Formación Doán desde aproximadamente los 
$1700 \mathrm{~m}$ hasta $1400 \mathrm{~m}$, y a partir de ese punto el descenso del río es más suave donde inician los Depósitos no Consolidados.

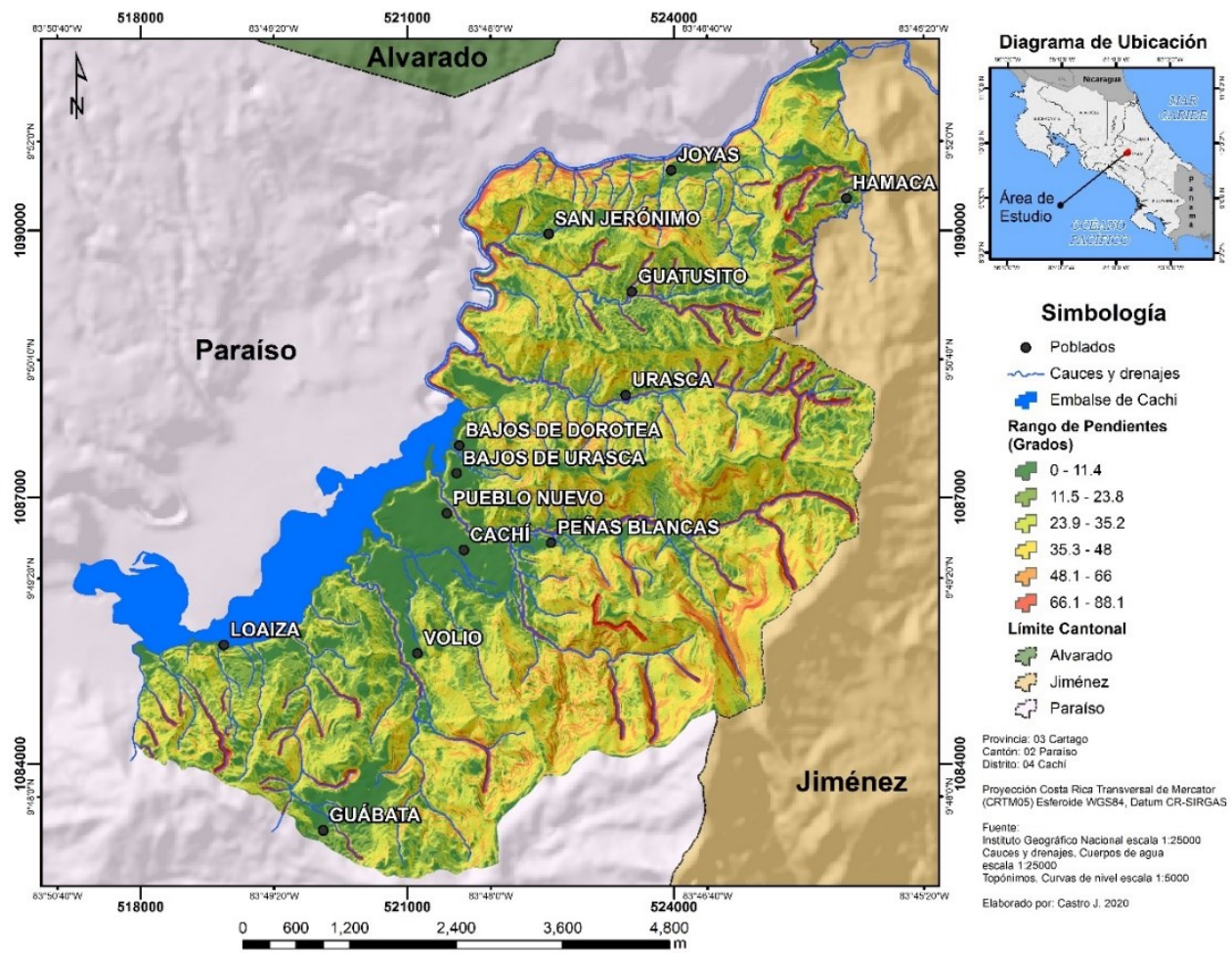

Fig. 2. Modelo de pendientes del terreno en grados del distrito de Cachí

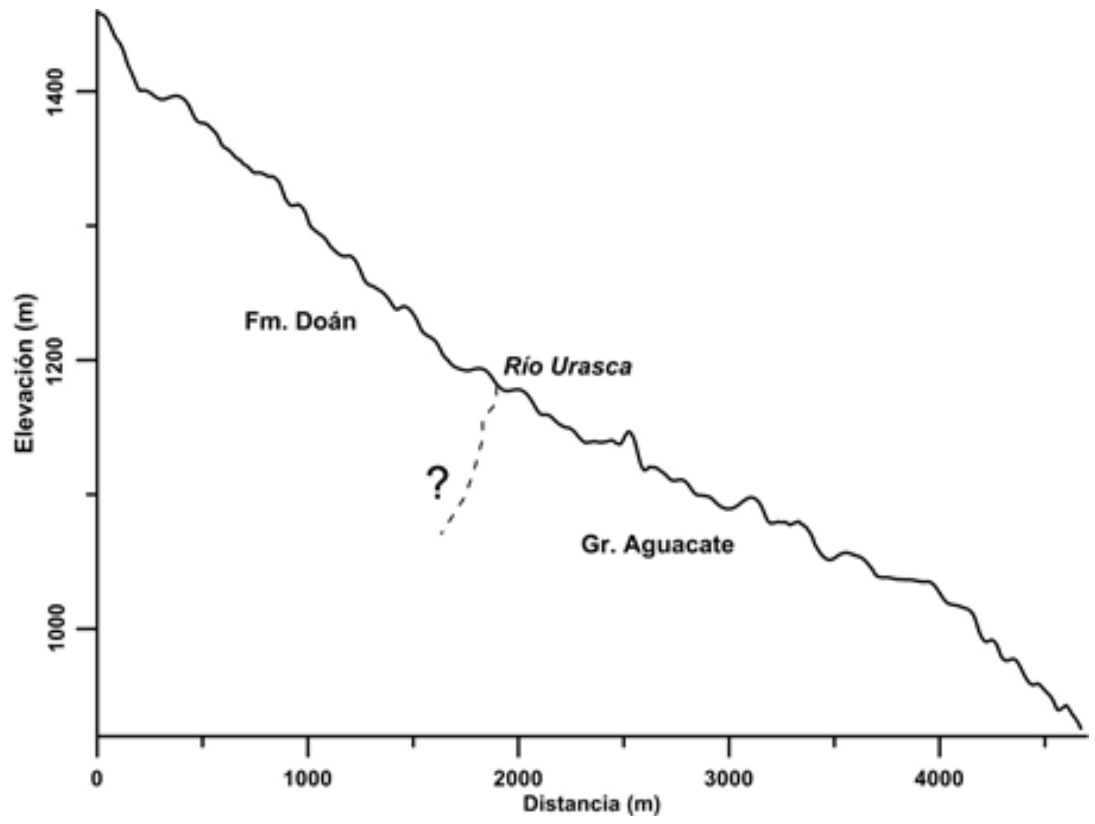

Fig. 3. Perfil longitudinal del río Urasca en el distrito de Cachí 


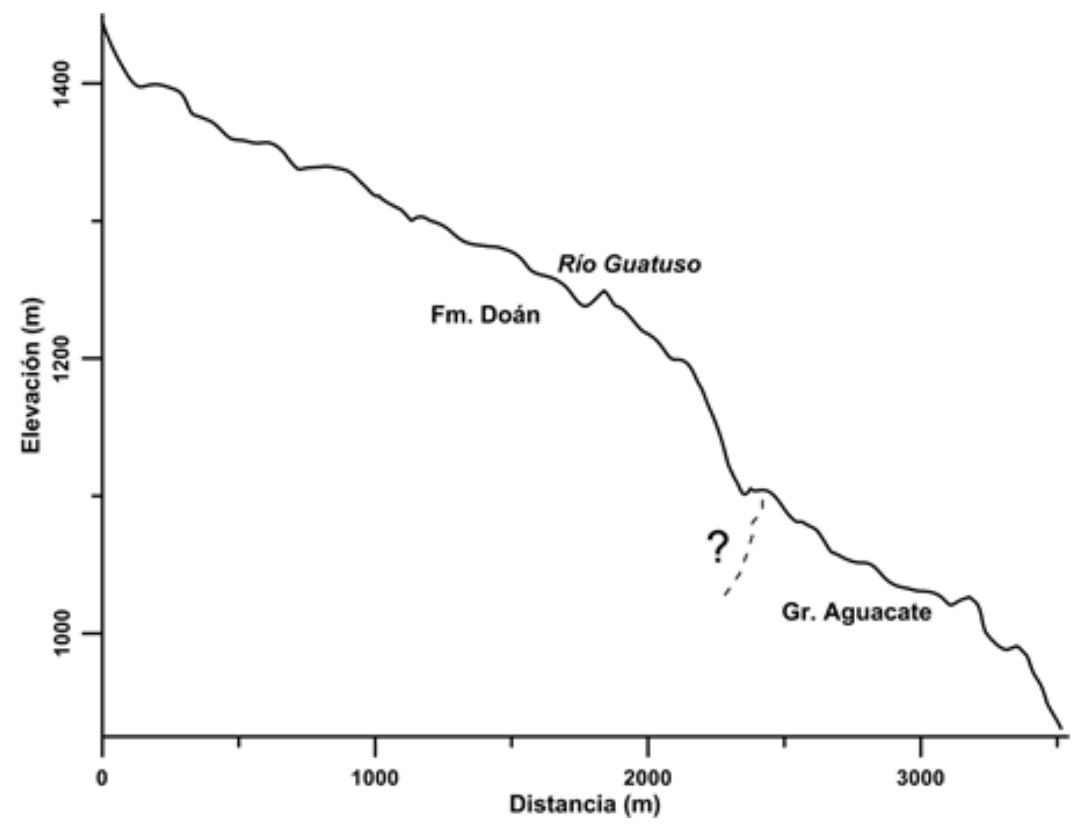

Fig. 4. Perfil longitudinal del río Guatuso en el distrito de Cachí

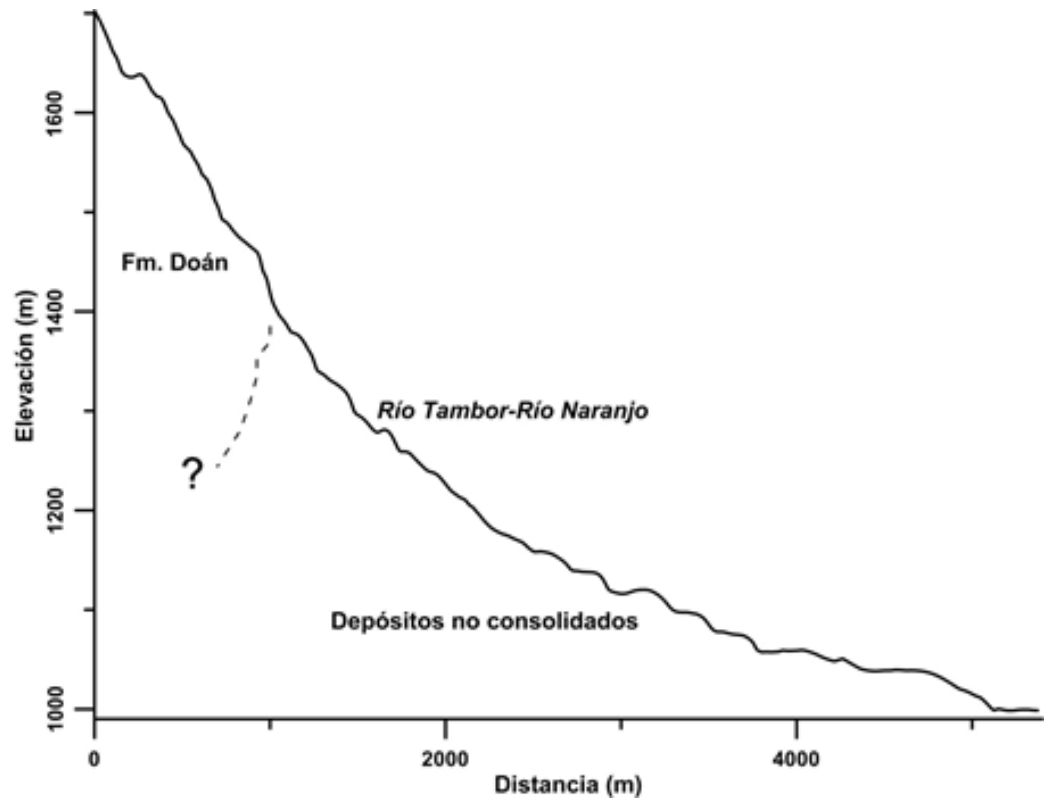

Fig. 5. Perfil longitudinal del río Naranjo en el distrito de Cachí

Los principales drenajes del distrito son perpendiculares al río Reventazón con una dirección sureste-noroeste proveniente de la parte montañosa del distrito donde se ubica el cerro Doán (Fig. 6.).

Unidades hidrogeológicas: La figura 7 presenta las principales unidades geológicas distribuidas en el área de estudio: formaciones Pacacua, Aguacate, Doán, Ujarrás, La Cruz, Toba de San Jerónimo y Depósitos Recientes o No Consolidados (Fig. 7.). 


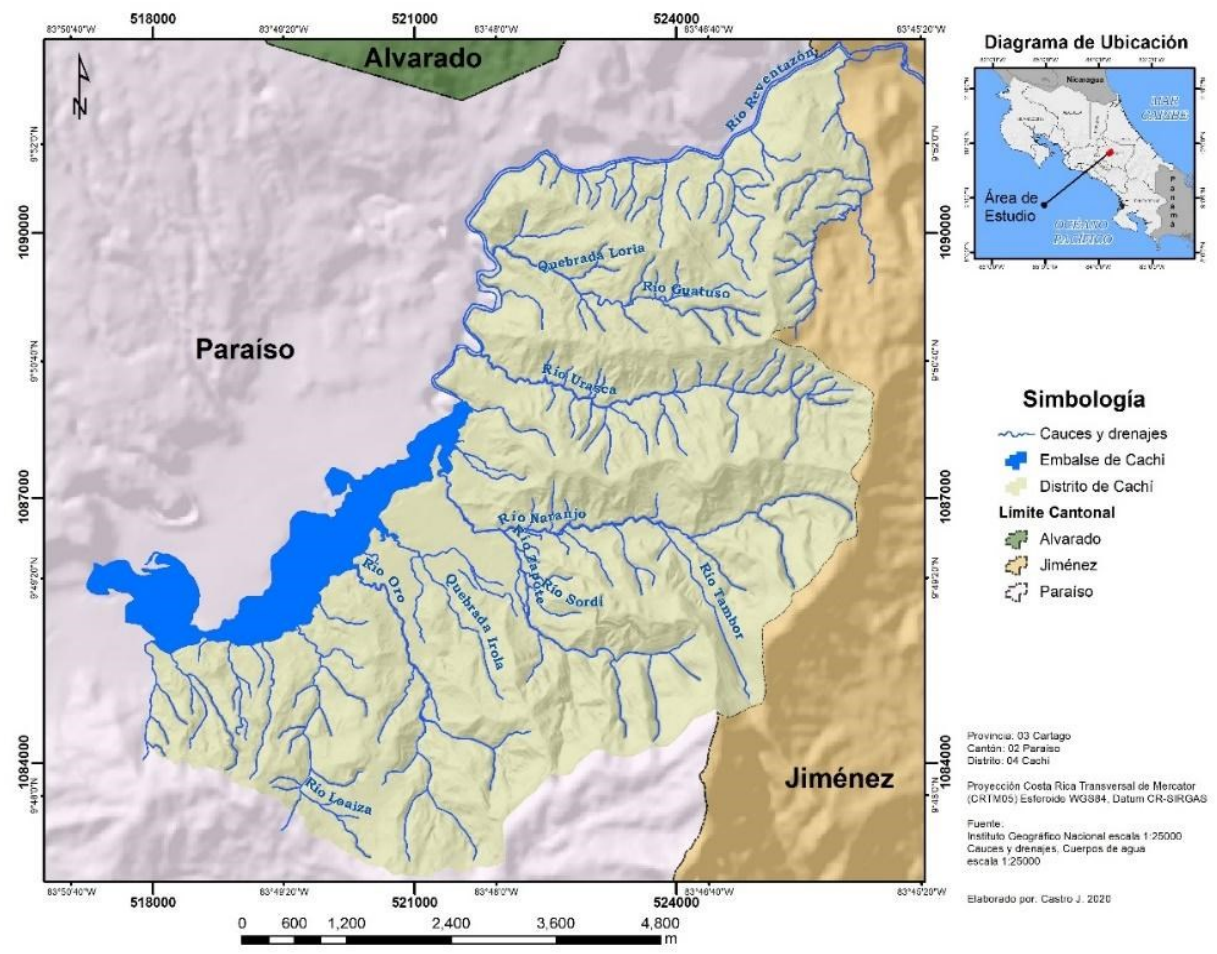

Fig. 6. Principales drenajes del distrito Cachí

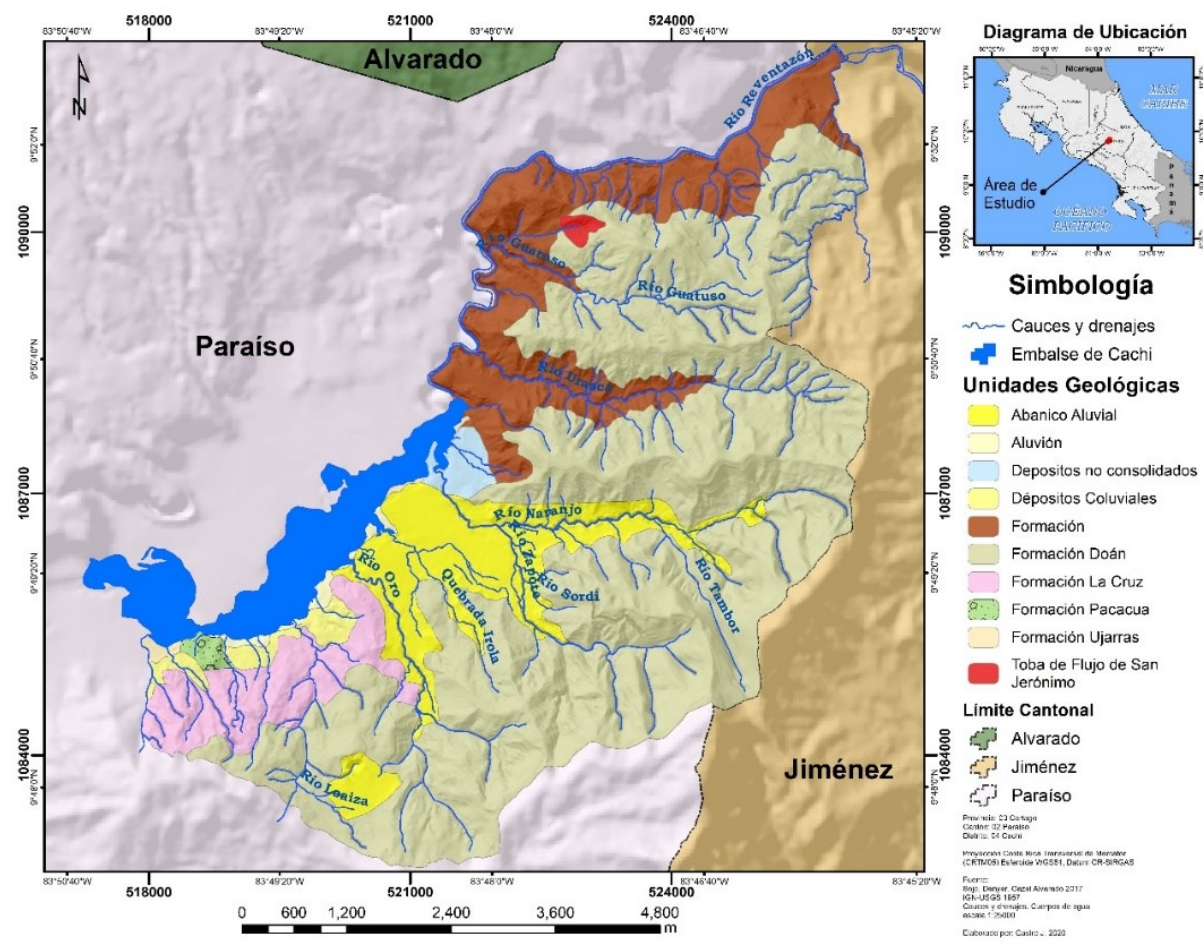

Fig. 7. Unidades geológicas del distrito de Cachí 
A partir de ellas se definieron cuatro unidades hidrogeológicas que poseen relación con los manantiales del área en estudio (Fig. 8.):

a) Unidad Cachí: conformada por depósitos no consolidados de origen fluvial y lacustre ubicado en las zonas planas, los materiales fluviales son producto de la hidrodinámica de los ríos Oro, Zapote-Irola y Tambor-Naranjo, así como las terrazas formadas por la dinámica del río Reventazón, sin embargo, debido a la carencia de manantiales y pozos no se puede evidenciar esto totalmente.

b) Unidad Peñas Blancas: compuesto por productos volcánicos como flujos de lava, depósitos vulcanoclásticos de grano fino y grueso y tobas, los materiales de esta unidad corresponden con la Formación Doán. Aflora en la parte alta del territorio de Cachí, y se caracteriza por tener matriz fracturada, la cual origina las descargas por manantiales. Algunos manantiales son Palmichal, Guatusito, Lomo de Burro, San Jerónimo, Urasca 1 y 2, Jorge Obando, Nicanor, Chilamate 1 y 2 y Volio 1 y 2.

c) Unidad Loaiza: compuesta por materiales son vulcanoclastitas, dichos materiales probablemente conforman secuencias entre las cuales se puede generar la acumulación de agua, formando de esta manera un acuitardo o acuicludo. En el caso del manantial Loáiza, su surgencia podría explicarse por ciertas condiciones muy locales o por condiciones de fallamiento que hayan afectado estructuralmente a los materiales. Vargas \& Mora (1999), describen la producción de manantiales en los materiales de la formación Pacacua, lo cual podría explicar la surgencia del manantial Loáiza en este tipo de materiales.

d) Unidad San Jerónimo: conformada por materiales volcánicos que forman parte de la ribera derecha del río Reventazón en el límite norte y noroeste del distrito de Cachí y que corresponden con la Formación Aguacate.

Propiedades físicas e hidráulicas de los materiales: En promedio los porcentajes de arcilla fueron de $29,9 \%, 27,6 \%$ de limo y $42,7 \%$ de arena. En la parte alta las retenciones fueron menores debido a la presencia de granulometrías más gruesas. En el sector este, donde hubo presencia de depósitos superficiales se originaron texturas más gruesas, debido al encontrarse arenas depositadas por los sistemas hidrográficos Tambor-Naranjo, Zapote-Irola y Oro. Respecto a la densidad aparente, esta oscila de 1,08 a $1,22 \mathrm{~g} / \mathrm{cm}^{3}$ y la densidad real de partículas se encuentra entre 2,06 a $2,32 \mathrm{gm} / \mathrm{cm}^{3}$. La conductividad hidráulica en las zonas del cerro Doán y poblado de Cachí muestran valores más altos, lo cual coincide con los valores menores de arcilla existentes en estos sitios. Es así que la retención de agua en el suelo para el distrito de Cachí mostró valores distribuidos en todas las microcuencas con un promedio de $37,5 \%$ y $21,7 \%$ para los valores máximo y mínimo de retención de humedad, respectivamente. Este factor es significativo ya que parte del sector se dedica a actividades agropecuarias.

Uso de la tierra: La distribución es muy variable, aproximadamente un $50 \%$ tiene cobertura boscosa en la parte alta lo cual coincide con las zonas donde se encuentran los manantiales. Las actividades agropecuarias representan un $39,3 \%$ ubicados en las partes medias y bajas de las cuencas que lo conforman (Tabla 1).

Se reconoce un desarrollo importante de cultivos aproximadamente $26 \%$ en la parte Sur en los poblados Loaiza, Volio, Peñas Blancas y Cachí, en su mayoría por el cultivo de café. Así mismo en el sector de Guatuso y sus alrededores se dedican a la actividad pecuaria (Fig. 9.). 


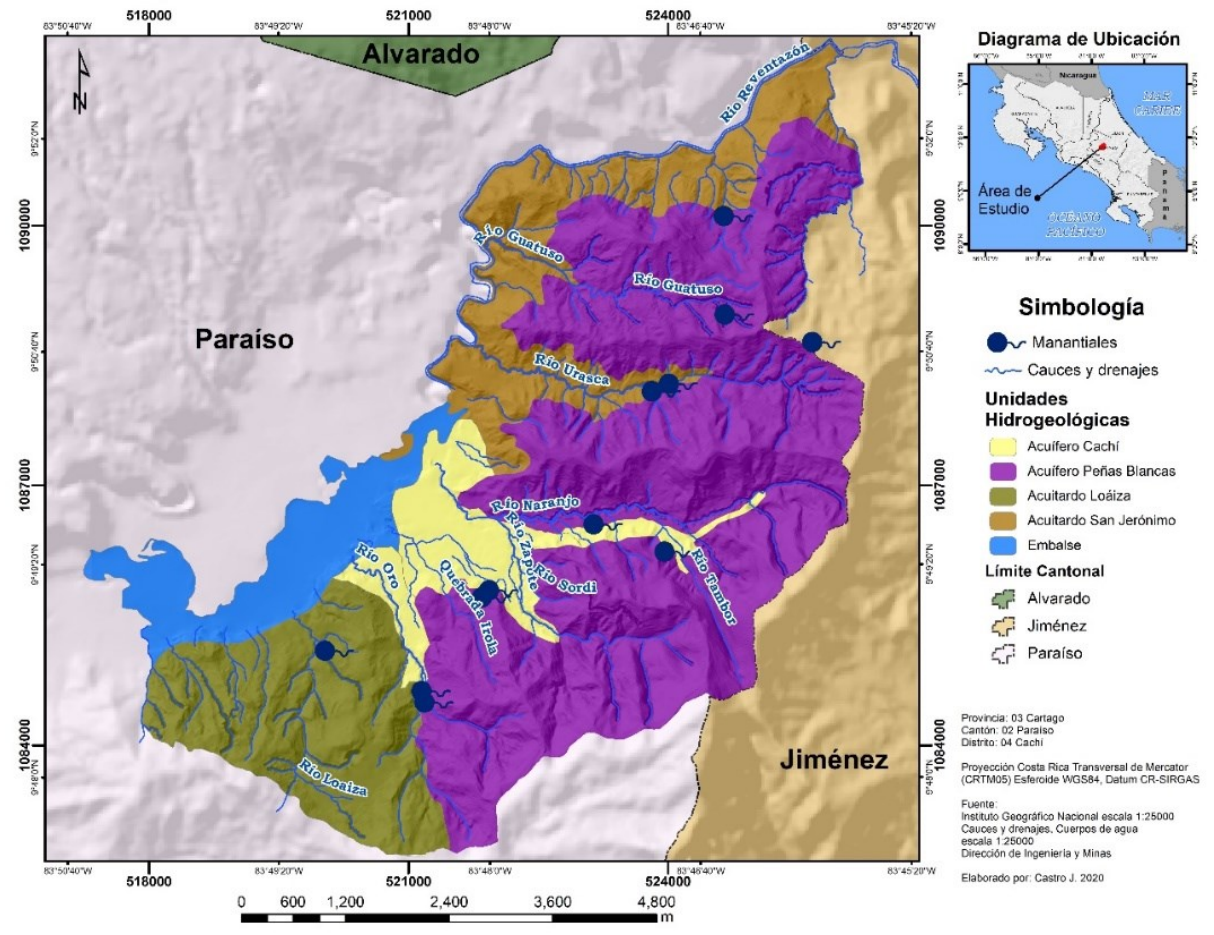

Fig. 8. Unidades hidrogeológicas del distrito de Cachí

TABLA 1

Clasificación del uso de la tierra en las microcuencas del distrito de Cachí

\begin{tabular}{lcc}
\hline \multicolumn{1}{c}{ Uso } & $\begin{array}{c}\text { Área } \\
\left(\mathbf{K m}^{\mathbf{2}}\right)\end{array}$ & $\begin{array}{c}\text { Uso de la Tierra } \\
(\mathbf{\%})\end{array}$ \\
\hline Bosque & 20,69 & 50,2 \\
Cultivo & 10,67 & 25,9 \\
Pastos & 5,51 & 13,4 \\
Cuerpos de agua & 2,84 & 6,9 \\
Urbano & 0,96 & 2,3 \\
Suelo descubierto & 0,54 & 1,3 \\
Total & $\mathbf{4 1 , 2 4}$ & $\mathbf{1 0 0}$ \\
\hline
\end{tabular}

Balance hídrico de suelos: Con base en la lluvia zonal, las pendientes y el uso de la tierra se determinaron 19 zonas hidrológicas (Fig. 10.).

Los resultados del balance hídrico de suelos mostraron zonas de recarga en el sector de San Jerónimo, Urasca, Cachi, Guatusito, Peñas Blancas y Volio con valores que oscilan de $792-796 \mathrm{~mm}$, estos sitios coinciden con el uso de cultivos y pastos (Fig. 11.). 


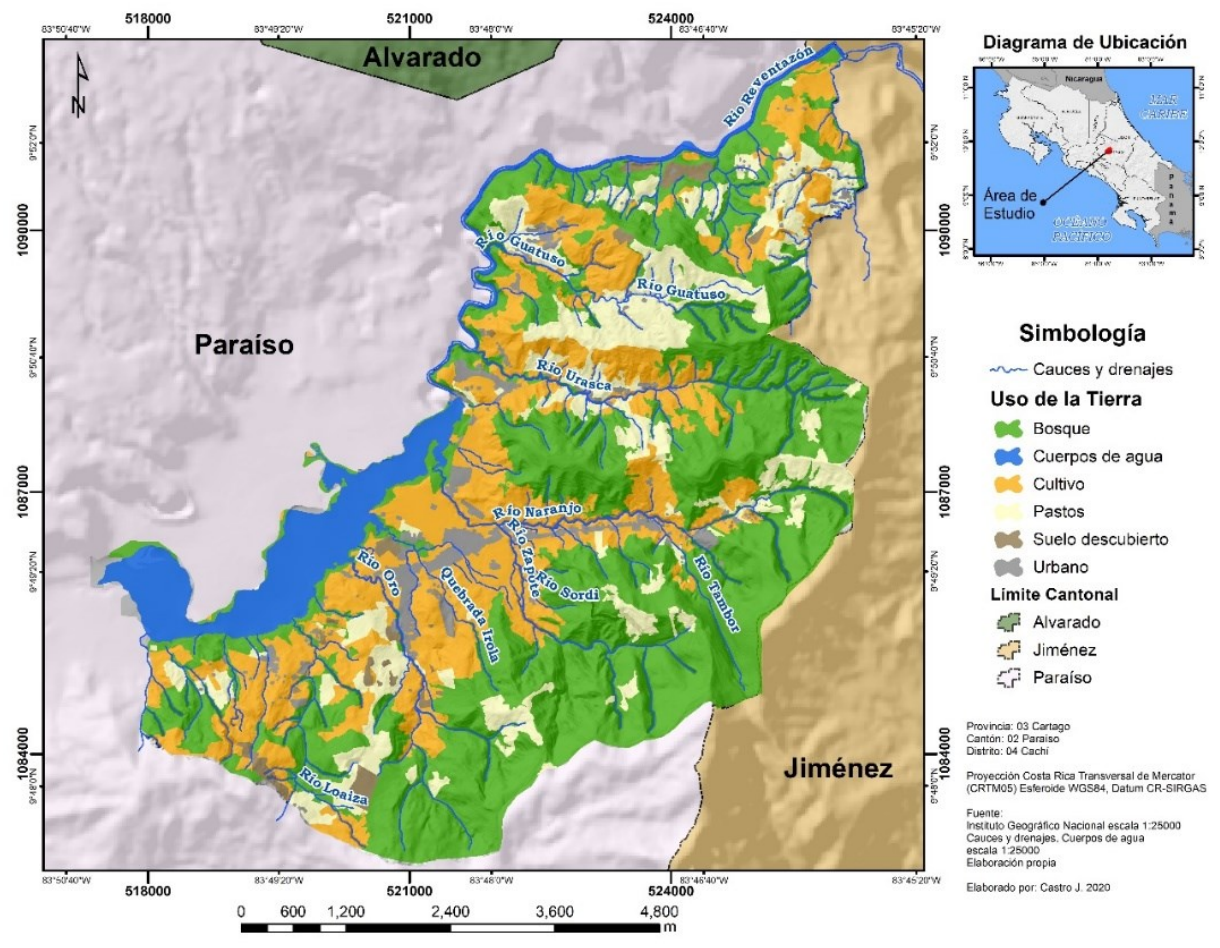

Fig. 9. Uso de la tierra en el distrito de Cachí

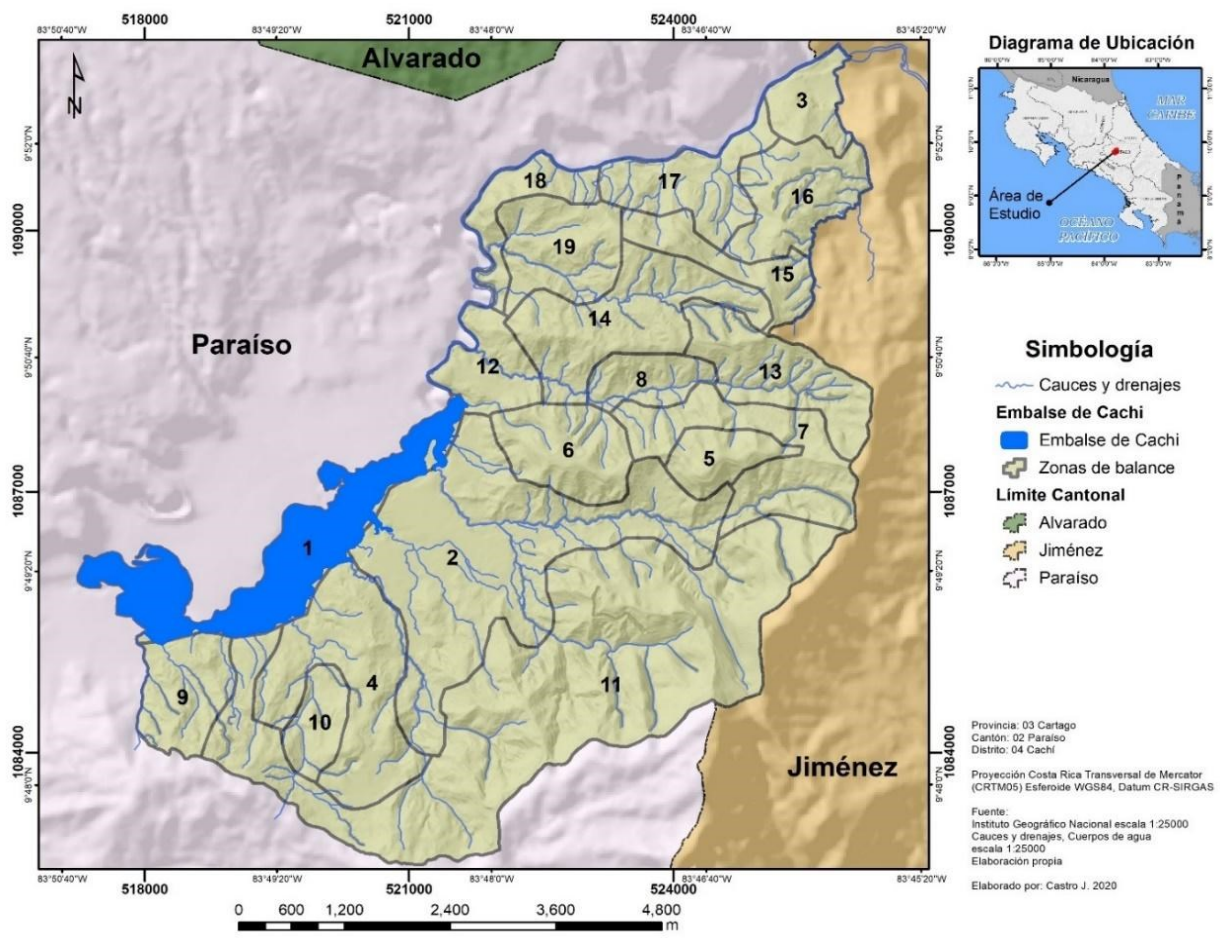

Fig. 10. Zonas hidrológicas pertenecientes al distrito de Cachí, Cartago 


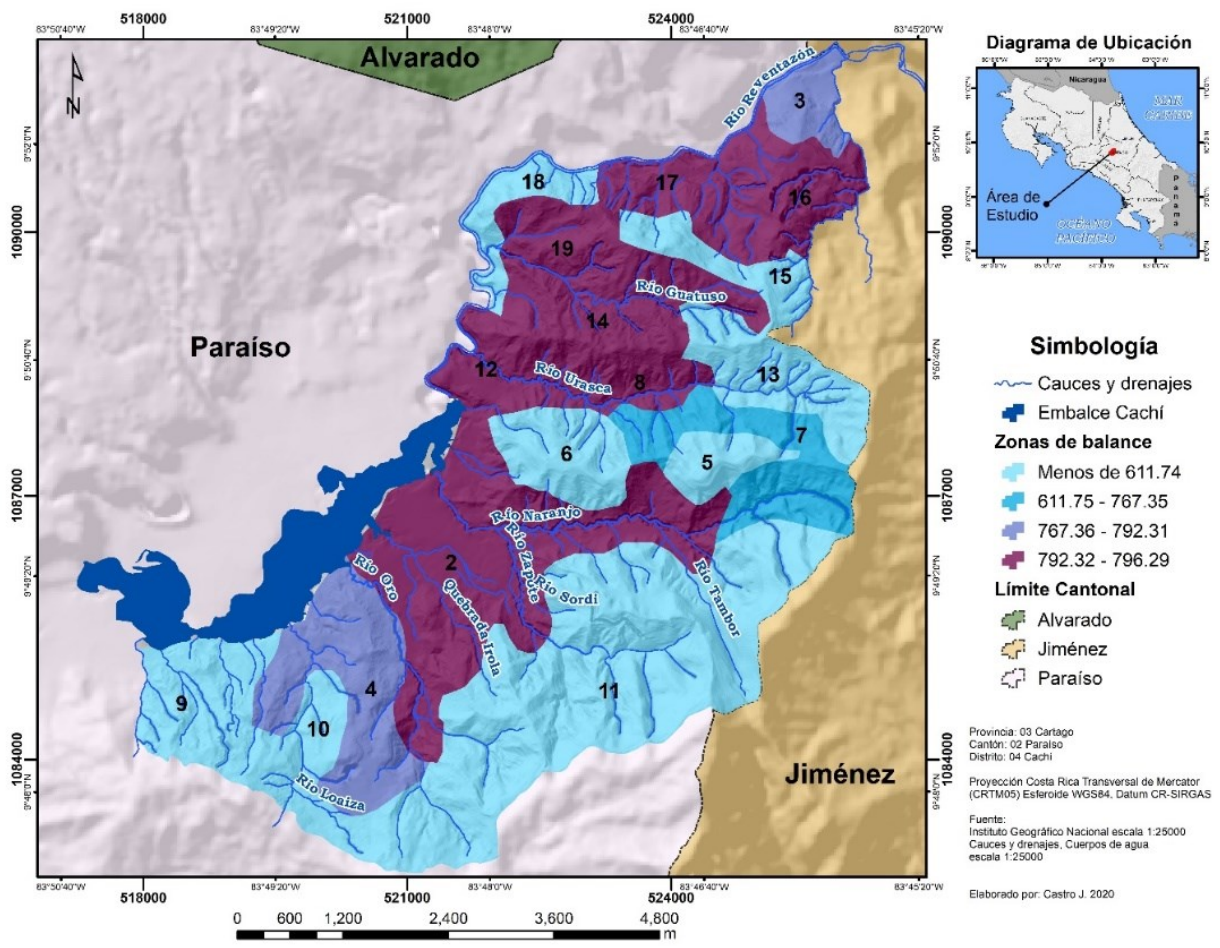

Fig. 11. Zonas de recarga potencial en las microcuencas del distrito de Cachí, Cartago

\section{DISCUSIÓN}

En el sector de la Formación Doán, el mayor grado de inclinación del terreno podría propiciar una mayor escorrentía y disminuir la recarga potencial, a diferencia del sector suroeste, cerca del poblado de Loáiza, donde la pendiente es menor. Esto se explica por el hecho de que habrá un menor tiempo de encharcamiento del agua en el terreno y una mayor energía de flujo de agua favorecido por la inclinación. Otros autores han señalado el efecto de la pendiente en la infiltración del terreno (Harden \& Scruggs, 2003).

El perfil longitudinal del río Urasca presentó una tendencia hacia una forma convexa, que facilita el transporte de sedimentos y la erosión del lecho fluvial (Hack, 1957), además se presenta hacia un estado normal de equilibrio dinámico (Snow \& Slingerland, 1987; Whipple \& Tucker, 1999; Whipple et al., 2011). Al inicio del perfil, el gradiente altitudinal es menor por lo que aumenta su pendiente, conforme se avanza en el trayecto disminuye la altitud, por tanto, la diferencia altimétrica con respecto a la distancia se incrementa, disminuyendo su pendiente.

Según Vargas \& Mora (1999), la producción de manantiales en los materiales de la Formación Pacacua, podría explicar la surgencia del manantial Loáiza en este tipo de materiales. La unidad presenta buenas condiciones hidrogeológicas porque permite relacionar la conexión hidráulica con las unidades existentes. Los manantiales muestran una dirección del agua subterránea muy relacionada con la topografía dominante de la zona, en especial con la distribución de las unidades hidrogeológicas, con una dirección preferencial de flujo noroeste (NW) desde las partes altas de la unidad hidrogeológica Peñas Blancas que corresponden con los cerros Doán. La superficie piezométrica regional mostró una posible división del agua subterránea cerca del manantial Guatusito donde el agua fluye por la disminución de su gradiente hidráulico, de esta manera puede existir un aporte hidrogeológico hacia las cuencas de los sistemas hidrográficos Guatuso y Urasca. 
Chicas et al. (2014) señalan que es necesario mantener niveles adecuados de agua en el suelo para garantizar el éxito en los cultivos. Se ha identificado un considerable desarrollo del suelo en todo el distrito, que favorece la retención de humedad en espacio porosos donde en promedio los porcentajes de arcilla es de $29,9 \%, 27,6 \%$ de limo y de $4,7 \%$ de arena. Sandoval (2007) menciona que la capacidad de retención de humedad tiene una estrecha relación con la textura de los suelos, para el caso del distrito, aproximadamente un $30 \%$, corresponde a la presencia de suelos arcillosos y el restante representa granulometría de media o gruesa.

Bras (1990) afirma que la columna de agua en suelos con un mayor porcentaje de arcillas puede llegar a contener 10 veces más agua retenida que un suelo arenoso, otros como Rawls et al. (1993) indican que es mayor la capacidad de retención en suelos con un contenido de arcilla mayor a 10\%, lo que puede representar parte de la zona de Cachí. Los pequeños espacios porosos del suelo hacen posible que un mayor volumen de agua se retenga en el suelo favoreciendo así la recarga de aguas subterráneas (Rojas, 2010).

En la parte alta, las menores retenciones de agua debido a la presencia de granulometrías más gruesas y en el sector de Cachí, la mayor presencia de depósitos superficiales, indican la relación entre la retención de humedad y el tamaño de grano, esta relación ha sido también encontrada por otros autores (Jabro et al., 2009); lo cuales han indicado la dependencia de la curva de retención con respecto a la distribución de las partículas (Shwetha \& Varija, 2015).

En cuanto a la densidad aparente los cambios en esta variable están directamente vinculadas con los usos de la tierra (Shan et al., 2019) estos pueden estar relacionados a las variaciones en los espacios porosos (Assouline, 2006). En lo que respecta a la conductividad hidráulica en su mayoría la variación está vinculada con las fracciones texturales (Nath \& Krishna, 2014) y con los cambios de uso de la tierra (Neris et al., 2012). La relación del uso de la tierra y la conductividad hidráulica podría estar atribuida a la porosidad total del suelo (Sun et al., 2018)

En el distrito de Cachi los distintos usos de la tierra, los afloramientos litológicos y la topografía del terreno están relacionados a la dinámica de la recarga potencial (Seyfried \& Wilcox, 2006; Min et al., 2015) a partir de la infiltración de la lluvia y su relación con los acuíferos (Nemaxwi et al., 2019).

Las zonas de captura y de protección de los manantiales están íntimamente ligadas a las variaciones de uso de la tierra (López et al., 2021) debido a que esto podría modificar las tasas de recarga y el transporte de contaminantes (Scalon et al., 2005) desde la superficie hasta el nivel piezométrico.

El factor del uso de la tierra es el que tiene mayor variabilidad en la determinación de la recarga potencial (Walker et al., 2005). De la misma forma, las variaciones en la evapotranspiración pueden variar de acuerdo con el uso de la tierra (Li et al., 2021).

\section{AGRADECIMIENTOS}

Agradecemos al proyecto B1402 del Centro de Investigaciones en Ciencias Geológicas de la Universidad de Costa Rica y proyecto 0114-19 de la Escuela de Ciencias Ambientales de la Universidad Nacional de Costa Rica. 


\section{ÉTICA, CONFLICTO DE INTERESES Y DECLARACIÓN DE FINANCIAMIENTO}

Declaramos haber cumplido con todos los requisitos éticos y legales pertinentes, tanto durante el estudio como en la preparación de este documento; que no hay conflictos de interés de ningún tipo, y que todas las fuentes financieras se detallan plena y claramente en la sección de agradecimientos. Asimismo, estamos de acuerdo con la versión editada final de esta publicación. El respectivo documento legal firmado se encuentra en los archivos de la revista.

La declaración de contribución de cada autor es la siguiente: P.R.G: Trabajo de campo, recolección de muestras, modelado de datos, recolección de datos, P.R.G., M.A.J y D.S.T: análisis de datos y revisión de las referencias. J.C.S: elaboración de los mapas. Todos los coautores.: preparación y aprobación final del manuscrito.

\section{REFERENCIAS}

Achu, A., Thomas, J., \& Reghunath, R. (2020). Multi-criteria decision analysis for delineation of groundwater potential zones in a tropical river basin using remote sensing, GIS and analytical hierarchy process (AHP). Groundwater for Sustainable Development, 10, 100365. https://doi.org/10.1016/j.gsd.2020.100365

Acuña R. (1 de febrero de 2008). Sistema Nacional de Información Territorial. Curvas de nivel cada 10 metros 1:5000 Costa Rica. https://bit.ly/3FUuVWV

Acuña R. (14 de mayo de 2014). Sistema Nacional de Información Territorial. Cauce y Drenaje a escala 1:25000 Costa Rica. https://bit.ly/3xuOKBd

Assouline, S. (2006). Modeling the relationship between soil bulk density and the water retention curve. Vadose Zone Journal, 5(2), 554-563. https://doi.org/10.2136/vzj2005.0083

Bras, R. (1990). Hydrology: An Introduction to Hydrologic Science. Addison Wesley Publishing Company.

Chicas, A., Vanegas, E., \& García. N. (2014). Determinación indirecta de la capacidad de retención de humedad en suelos de la subcuenca del río Torjá, Chiquimula, Guatemala. Revista Ciencias Técnicas Agropecuarias, 23(1), 41-46.

Cortés, V. (2008). Discrepancias entre uso actual y potencial de las laderas del Valle de Orosi. Revista Reflexiones,87(2), 91-110. https://revistas.ucr.ac.cr/index.php/reflexiones/article/view/11498

Dressler, M. (2009). Art of Surface Interpolation.Technical University of Liberec.

Hack, J. (1957). Studies of Longitudinal Stream Profiles in Virginia and Maryland. US Government Printing Office. https://doi.org/10.3133/pp294B

Hall, B., Currell, M., \& Webb, J. (2020). Using multiple lines of evidence to map groundwater recharge in a rapidly urbanising catchment: Implications for future land and water management. Journal of Hydrology, 580, 124265. https://doi.org/10.1016/j.jhydrol.2019.124265

Harden, C. P., \& Scruggs, P. D. (2003). Infiltration on mountain slopes: a comparison of three environments. Geomorphology, 55(1-4), 5-24. https://doi.org/10.1016/S0169-555X(03)00129-6

Hutchinson, M. F. (1993). Development of a continent-wide DEM with applications to terrain and climate analysis. In M. Goodchild, B.O. Parks, \& L. Steyaert (Eds.), Environmental Modeling with GIS (p. 392-399). Oxford University Press.

Instituto Costarricense de Turismo (ICT). (2002). Plan de Desarrollo Turístico de Unidad Valle Central del Sector de Cartago. https://bit.ly/3lfKhNS 
Jabro, J., Evans, R., Kim, Y., \& Iversen, W. (2009). Estimating in situ soil-water retention and field water capacity in two contrasting soil textures. Irrigation Science, 27, 223-229. https://doi.org/10.1007/s00271-008-0137-9

Kundzewicz, Z, \& Döll, P. (2009). Will groundwater ease freshwater stress under climate change? Hydrological Sciences Journal, 54(4), 665-675. https://doi.org/10.1623/hysj.54.4.665

Li, M., Chu, R., Islam, A., Shen, S. (2021). Characteristics of surface evapotranspiration and its response to climate and land use and land cover in the Huai River Basin of eastern China. Environmental Science and Pollution Research, 28, 683-699. https://doi.org/10.1007/s11356-020-10432-9

López, S.; Expósito, J.; Esteller, M.; Gómez, M., Paredes, J.; \& Esquivel, J. (2021). Delineation of protection zones for springs in fracture volcanic media considering land use and climate changes scenarios in Central Mexico region. Environmental Earth Sciences, 80, 366. https://doi.org/10.1007/s12665-021-09662-y

Min, L., Shen, Y., \& Pei, H. (2015). Estimating groundwater recharge using deep vadose data under typical irrigated cropland in the piedmont region of the North China Plain. Journal of Hydrology, 527: 305-315. https://doi.org/10.1016/j.jhydrol.2015.04.064

Nath, T., \& Krishna, B. (2014). Influence of soil texture and total organic matter content on soil hydraulic conductivity of some selected tea growing soils in Dibrugarh district of Assam, India. International Research Journal of Chemistry and Chemical Sciences, 1(1), 002-009.

Nemaxwi, P., Odiyo, J., \& Makungo, R. (2019). Estimating of groundwater recharge response from rainfalls events in a semi-arid fractured aquifer: Casa study of quaternary catchmmen A91H, Limpopo Province. South Africa. Cogent Engineering, 6(1). https://doi.org/10.1080/23311916.2019.1635815

Neris, J., Jiménez, C., Fuentes, J., Morillas, M., \& Tejedor, M. (2012). Vegetation and land-use effects on soil properties and water infiltration of Andisols in Tenerife (Canary Islands, Spain). Catena, 98, 55-62. https://doi.org/10.1016/j.catena.2012.06.006

Planificación Regional y Urbana de la Gran Área Metropolitana (PRUGAM). (2007). Atlas Cartográfico Plan PRUGAM 20082030. MIVAH-MOPT-MIDEPLAN-MINSALUD-IVU-IFAM-CNFL-AYA

Rawls, W., Ahuja, L., Brakensiek, D., \& Shirmohammadi, A. (1993). Infiltration and Soil Water Movement. In D. Maidment (Ed), Handbook of Hidrology (pp. 1-51). McGraw-Hill.

Rojas, H. (2010). Áreas de recarga hídrica de la parte media-alta de las microcuencas Palo, Marín y San Rafaelito, San Carlos, Costa Rica. UNED Research Journal, 2(2), 181-204.

Sandoval, J. (2007). Principios de Riego y Drenaje. Editorial Universitaria de la Universidad de San Carlos de Guatemala.

Scalon, B., Reedy, R., Stonestrom, D., Prudic, D., \& Dennehy, K. (2005). Impact of land use and land cover change on groundwater recharge and quality in the southwestern US. Global Change Biology, 11(10), 1577-1593. https://doi.org/10.1111/j.1365-2486.2005.01026.x

Schosinsky, G. (2006). Cálculo de la recarga potencial de acuíferos mediante un balance hídrico de suelos. Revista Geológica de América Central, 34-35, 13-30. https://doi.org/10.15517/rgac.v0i34-35.4223

Schosinsky, G., \& Losilla, M. (2000). Modelo analítico para determinar la infiltración con base en la lluvia mensual. Revista Geológica de América Central, 23, 43-55.

Seyfried, M., \& Wilcox, B. (2006). Soil water storage and rooting depth: Key factors controlling recharge on rangelands. Hydrological Processes, 20(15), 3261-3275. https://doi.org/10.1002/hyp.6331

Shan, L; Qi-quan, L; Chang-quan; W; Bing; L; Xue-song; G; Yi-ding, L; De-yon, W. (2019). Spatial variability of soil bulk density and its controlling factors in and agricultural intensive area of Chengdu Plain, Southwest China. Journal of Integrative Agricultura, 18(2), 290-300. https://doi.org/10.1016/S2095-3119(18)61930-6 
Shwetha, P., \& Varija, K. (2015). Soil water retention curve from saturated hydraulic conductivity for sandy loam and loamy sand textured soils. Aquatic Procedia, 4, 1142-1149. https://doi.org/10.1016/j.aqpro.2015.02.145

Snow, R., \& Slingerland, R. (1987). Mathematical modeling of graded river profiles. The Journal of Geology,95(1), 15-33. https://doi.org/10.1086/629104

Sojo, D., Denyer, P., Gazel, E., \& Alvarado, G. (2017). Geología del cuadrante Tapantí (1: 50 000), Costa Rica. Revista Geológica de América Central, (56), 83-116. https://doi.org/10.15517/rgac.v0i56.29238

Sun, D., Yang, H, Guan, D., Yang, M., Wu, J., Yuan, F., Jin, C., Wang, A., \& Zhang, Y. (2018). The effects of land use change on soil infiltration capacity in China: A meta-analysis. Science of the Total Environment, 626, 1394-1401. https://doi.org/10.1016/j.scitotenv.2018.01.104

Vargas, A., \& Mora, R. (1999). Hidrogeoquímica y producción de manantiales en las formaciones Pacacua y Peña Negra. Revista Geológica de América Central, 22, 101-111.

Varol, S., \& Davraz, A. (2015). Evaluation of the groundwater quality with WQI (Water Quality Index) and multivariate analysis: a case study of the Tefenniplain (Burdur/Turkey). Environmental Earth Sciences,73(4), 1725-1744. https://doi.org/10.1007/s12665-014-3531-z

Vélez, M., \& Vásquez, L. (2004). Métodos para determinar la recarga en acuíferos. Avances en recursos hidráulicos, (11), 51-62.

Walker, G., Zhang, L., Ellis, T., Hatton, T., \& Petheram, C. (2005). Estimating impacts of changed land use on recharge: Review of modelling and other approaches appropriate for management of dryland salinity. Hydrogeology Journal, 10, 68-90. https://doi.org/10.1007/s10040-001-0181-5

Whipple, K., \& Tucker, G. (1999). Dynamics of the stream-power river incision model: Implications for height limits of mountain ranges, landscape response timescales, and research needs. Journal of Geophysical Research: Solid Earth, 104(B8), 17661-17674. https://doi.org/10.1029/1999JB900120

Whipple, K., DiBiase, R., \& Crosby, B. (2011). Bedrock Rivers. In J. Shroder (Ed.), Treatise on Geomorphology (p. 550-573). Academic Press. 